Supplemental Information

\title{
Evaluation of sampling, analysis, and normalization methods for SARS-CoV-2 concentrations in wastewater to assess COVID-19 burdens in Wisconsin communities
}

Shuchen Feng ${ }^{* 1+}{ }^{\dagger}$, Adélaïde Roguet ${ }^{* 1}$, Jill S. McClary-Gutierrez ${ }^{1 \ddagger}$, Ryan J. Newton ${ }^{1}$, Nathan Kloczko², Jonathan G. Meiman², Sandra L. McLellan ${ }^{1 * *}$

${ }^{1}$ School of Freshwater Sciences, University of Wisconsin-Milwaukee, Milwaukee, Wisconsin, 53204, USA

${ }^{2}$ Department of Health Services, Division of Public Health, Bureau of Environmental and Occupational Health, Madison, Wisconsin, 53703, USA

*Contributed equally to this work

**Corresponding author

${ }^{\dagger}$ Current address: Department of Civil \& Environmental Engineering, Northwestern University, Evanston, Illinois, 60208, USA

${ }^{\ddagger}$ Current address: Department of Civil \& Environmental Engineering \& Earth Sciences, University of Notre Dame, Notre Dame, Indiana, 46556, USA

Number of pages: 15

Number of tables: 2

Number of figures: 8 


\section{List of Supplemental Datasets, Text, Tables, and Figures}

Supplemental Dataset 1 List of the samples and metadata (excel spreadsheet)

Supplemental Text 1 Materials and Methods supplements and optimizations

Supplemental Text 2 Comparison of direct extraction recoveries with HA filter recoveries

Supplemental Text 3 Details for statistical methods

Table S1 List of the ddPCR and qPCR assays

Table S2 Influence of the incubation time on BCoV recovery rates and N1 and N2 concentrations

Figure S1 Influence of BCoV incubation time (with or without shaking) on N1/N2 detection and BCoV recovery

Figure S2 Two-dimensional RT-ddPCR cluster plots of N1/N2 duplex assays using single- and double- quenched N2 probes

Figure S3 Nested analysis of variance results for assays, filters, samples, and WWTP

Figure S4 Distribution of (a) BCoV recovery rates and (b) PMMoV concentrations averaged per day and per WWTP

Figure S5 Relationship between the human marker (PMMoV and HF183) concentrations and WWTP average daily flow

Figure S6 Spearman's rank correlation coefficients between case rate per 100,000 people averaged per 7-day or 3-day and the N1 and N2 concentration in sewage with and without normalization

Figure S7 95\% confidence intervals for Spearman's rank coefficients between N1 wastewater concentrations and diagnosed COVID-19 clinical cases.

Figure S8 Correlation coefficients for +/- 7 days (lag) between testing data (by date of specimen collection) and load-normalized SARS-CoV-2 concentrations in sewersheds. 


\section{Supplemental Text 1: Materials and Methods supplements and optimizations}

Filtration and extraction protocols are available on protocols.io:

dx.doi.org/10.17504/protocols.io.bue9nth6 and dx.doi.org/10.17504/protocols.io.budtns6n, respectively.

Bovine coronavirus internal recovery control Bovine coronavirus (BCoV) solution was prepared out of one dose of freeze-dried Calf Guard cattle vaccine (Zoetis, Parsippany, NJ) rehydrated in $3 \mathrm{~mL}$ of sterile $1 \mathrm{X}$ TE buffer and aliquoted into $100 \mu \mathrm{L}$ stock solution aliquots, which were stored immediately at $-80^{\circ} \mathrm{C}$. To use, the BCoV stock solution aliquot was thawed on ice and vortexed thoroughly; each aliquot was used for a maximum of two freeze-thaw cycles. A volume of $5 \mu \mathrm{L}$ of the BCoV stock solution (about $100,000 \mathrm{cp} / \mu \mathrm{L}$ ) was spiked into each $50 \mathrm{~mL}$ tube containing $25 \mathrm{~mL}$ of sample prior to filtration.

To define the concentration of BCoV stock solution, we extracted in duplicate the RNA using the RNeasy PowerMicrobiome kit (Qiagen, Hilden, Germany) following manufacturer's instructions. The extracted BCoV RNA was then serially diluted 1:2 for four dilutions and run in duplicate using the BCoV assay [1] according to the one-step RT-ddPCR procedure as described in the main text. In samples, the $\mathrm{BCoV}$ recovery was calculated as the ratio of the final quantity measured to the quantity added to each wastewater sample and was used to estimate the loss of the RNA during sample processing. A detailed protocol of the BCoV solution preparation and the titer estimation is available at protocols.io: $\mathrm{dx}$.doi.org/10.17504/protocols.io.bue8nthw.

We observed that $\mathrm{BCoV}$ recoveries did not correlate to $\mathrm{N} 1$ or $\mathrm{N} 2$ recoveries when comparing the RSD of replicate filters (Spearman's rank correlation coefficients, BCoV, $n=62$, rho $=0.113$, see main text). Because $\mathrm{BCoV}$ is an artificial spike-in control, we examined if the time from spiking into a sample to processing that sample impacted its recovery since enveloped viruses are expected to adsorb onto solids in the wastewater matrix. We hypothesized that the lack of congruence between $\mathrm{BCoV}$ recoveries and $\mathrm{N} 1$ or $\mathrm{N} 2$ recoveries was a result of the longer time that SARS-CoV-2 interacted with the wastewater matrix. We found that different incubation times and agitation regimes had no effect on N1 and N2 recoveries (Figure S1). Results were confirmed across samples from several wastewater treatment plants (WWTPs) (Table S2).

Limits of blank, detection, and quantification For the N1 and N2 assays, we defined the limit of detection (LOD) as the lowest analyte concentration likely to be reliably distinguished from the limit of blank (LOB) and at which the detection is feasible, according to Armbruster and Pry, 2008 [2]. The LOB was determined by quantifying N1 and N2 in a pre-pandemic sewage influent sample collected in August 2019. The LOB was calculated as the maximum value from a $90 \%$ confidence interval of the average false positive droplets across 24 replicate measurements. If the LOB was zero (i.e., no positive droplet in any of the replicates), then the LOD was set at 3 positive droplets as a conservative threshold. Serial dilutions of the Exact Diagnostics SARS-CoV-2 standards (including $200 \mathrm{cp} / \mathrm{mL}$ of the N gene; Bio-Rad, Hercules, CA) were run in triplicates to determine the limit of quantification (LOQ). We define the LOQ as the lowest concentration at which the analyte can not only be reliably detected but also some 
predefined goals for bias and imprecision are met [2]. For N1 and N2 we set the LOQ as the concentration at which the relative standard deviation was $\leq 30 \%$ between triplicate assay measures and the difference between the calculated and expected concentrations was $\leq 30 \%$. The LOB, LOD, and LOQ were always rounded to the nearest higher integer. For both the onestep RT-ddPCR N1 and N2 assays, the LOD was set at 3 positive droplets (LOB was 0) and the LOQ was 10 positive droplets. This was applied to both N1 and N2 assays except for the N2 assay run with the single quenched probe (BHQ1), for which the LOB was 4 droplets, the LOD was 5 , and the LOQ was 9.

Inhibition control Bovine Respiratory Syncytial Virus (BRSV) Bovine Respiratory Syncytial Virus (BRSV) solution was prepared from 25 doses of freeze-dried Inforce 3 Cattle Vaccine (Zoetis, Kalamazoo, MI, USA) rehydrated in $5 \mathrm{~mL}$ of sterile $1 \mathrm{X}$ TE buffer and aliquoted into multiple 150 $\mu \mathrm{L}$ stock solution aliquots, which were stored immediately at $-80^{\circ} \mathrm{C}$. A total of $150 \mu \mathrm{L}$ were extracted using the RNeasy PowerMicrobiome kit as described for the direct extraction (see Supplemental Text 2). The extracted BRSV RNA was then serially diluted 1:10 in water for four dilutions and run in duplicate using the BRSV assay [3] according to the one-step RT-ddPCR procedure as described in the main text. Approximately 4000 copies of BRSV were spiked into each RT-ddPCR reaction to evaluate the potential inhibition of PCR amplification. BRSV was also spiked into two wells containing only sterile PCR grade water to serve as a no inhibition reference measure. No template control wells using PCR grade water were also included. Reaction inhibition in the wastewater samples was identified as the ratio of BRSV concentrations in the reference wells to the wastewater samples. If the BRSV concentration in the wastewater samples was $<50 \%$ of the reference wells, then the reaction was considered to be inhibited [4]. No inhibition was observed among the 75 wastewater samples tested (see Supplemental Dataset 1).

\section{Supplemental Text 2: Comparison of direct extraction recoveries with HA filter recoveries}

For some wastewater influent samples, results from virus concentration methods were compared to those from a direct extraction (i.e., no concentration step). The RNeasy PowerMicrobiome Kit (Qiagen, Hilden, Germany) was used to extract RNA for all direct extraction samples. To begin, 125 - $150 \mu \mathrm{L}$ of wastewater was transferred into a 2-mL tube containing $400 \mu \mathrm{L}$ of warmed PM1 solution. Four microliters of $\beta$-mercaptoethanol was then added to each tube. The mixture was vortexed 15 seconds, and then incubated at room temperature for $10 \mathrm{~min}$. The manufacturer's instructions were then followed, except that 100 $\mu \mathrm{L}$ of solution IRS was used. Sample RNA was eluted in $60 \mu \mathrm{L}$ of RNase-free water.

To assess the efficiency of our HA filtering method, we compared the recoveries of BCoV and pepper mild mottle virus (PMMoV) in samples that were processed with both HA filters and direct extraction. On average, the recoveries were correlated, but with lower recovery efficiency for BCoV on HA filters. The ratio of $\mathrm{HA}$ copies to direct extraction copies for BCoV was $7 \pm 4 \%$ (Spearman, $n=106$, rho $=0.003$ ) and $45 \pm 26 \%$ for PMMoV (Spearman, $n=106$, rho $=$ 0.383 ). The recovery efficiency of $\mathrm{N} 1$ and $\mathrm{N} 2$ could not be calculated owing to the low number of samples $(n=1)$ above the limit of quantification from the direct extraction. 


\section{Supplemental Text 3. Details for statistical methods}

A comparison between the N1 and N2 assay concentrations was performed only on samples where both assay concentrations were above the LOQ. To assess the importance of the assay, filter, and consecutive day sampling on SARS-CoV-2 measured concentration variation, we calculated the relative standard deviation (RSD) between replicate assays, replicate filters, and from samples collected over two consecutive days, respectively. We focused on Sunday and Monday samples because they represented $60 \%$ of our total day-to-day samples. Only quantifiable data, i.e., above the LOQ, were used in these analyses due to the higher variability associated with concentrations below the limit of quantification.

A nested variance components analysis (nested ANOVA) was performed on $\log _{10}$-transformed data from six WWTPs, where we had consecutive day samples processed in duplicate filters with each filter quantified in replicate assays, to evaluate the total variance explained by the assay, filter, sample and WWTP factors on the SARS-CoV-2 variability. The analysis was done using the function anovaVCA implemented in the R package VCA v1.4.3 [5]. This function equates observed ANOVA Type-I sums of squares to their expected values and solves the resulting system of linear equations for variance components. An ANOVA was also performed on these data using the function aov to examine the significance of individual factors in explaining the observed variability, as well as to check the normality of residuals (Figure S3).

One-way analysis of variance (ANOVA) followed by Tukey's post-hoc test was performed using the function aov to explore the difference of BCoV recovery rate and PMMoV and HF183 concentrations (used separately as dependent variables) across WWTPs (independent variable).

Spearman's rank correlation coefficients were determined using the functions cor.test or cor. The $95 \%$ interval confidence of the rho estimates was determined using the function SpearmanRho implemented in the R package DescTools v0.99.41 [6].

Monte Carlo simulations were used to evaluate the impacts of different sampling schemes on observed trends during the 3-week high frequency sampling period at the Milwaukee JI facility. For this analyses, averaged concentrations of N1 and N2 (designated as N1/N2) were leftcensored, that is, when all replicate/assays were below the LOD, the concentration was defined as LOD/2. Each day's SARS-CoV-2 N1 concentrations were represented by a uniform distribution between the minimum and maximum N1 concentrations detected across all filter and assay replicates for that date. The original dataset included biological duplicate samples for SMTWTh. Simulated datasets were then constructed to represent seven different sampling schemes: (1) SMTWTh without duplicates, and duplicate samples on (2) SMTW, (3) SMT, (4) SM, (5), S, (6) STTh, and (7) MW. Sampling schemes 2-5 cluster sampling dates near the beginning of the week, which is often logistically favorable to maximize weekday sample processing time after sample collection. For each simulation, values were randomly selected from the uniform distributions representing the appropriate sampling dates, a linear trend was fit to the data, and fit parameters were extracted. This was repeated 10,000 times for each of the seven sampling schemes. A linear trend was considered to be observed if the $p$-value of the slope was $<0.05$. 
Table S1 List of the RT-ddPCR and qPCR assays

\begin{tabular}{|c|c|c|c|c|c|}
\hline Assay & Sequence $5^{\prime}-3^{\prime}$ & Suppliers & PCR & $\begin{array}{l}\text { Annealing } \\
\text { temperature }\end{array}$ & Reference \\
\hline N1 & $\begin{array}{l}\text { F: GACCCCAAAATCAGCGAAAT } \\
\text { R: TCTGGTTACTGCCAGTTGAATCTG } \\
\text { P: } \\
\text { FAM/ACCCCGCAT/ZEN/TACGTTTGGTGGACC/IABkFQ }\end{array}$ & $\begin{array}{l}\text { Eurofins } \\
\text { Eurofins } \\
\text { IDT }\end{array}$ & $\begin{array}{l}\text { RT- } \\
\text { ddPCR }\end{array}$ & 55 & [7] \\
\hline N2 & $\begin{array}{l}\text { F: TTACAAACATTGGCCGCAAA } \\
\text { R: GCGCGACATTCCGAAGAA } \\
\text { P: HEX/ACAATTTGCCCCCAGCGCTTCAG/BHQ1 and } \\
\text { HEX/ACAATTTGC/ZEN/CCCCAGCGCTTCAG/IABkFQ }\end{array}$ & $\begin{array}{l}\text { Eurofins } \\
\text { Eurofins } \\
\text { IDT }\end{array}$ & $\begin{array}{l}\text { RT- } \\
\text { ddPCR }^{a}\end{array}$ & 55 & [7] \\
\hline $\mathrm{BCoV}$ & $\begin{array}{l}\text { F: CTGGAAGTTGGTGGAGTT } \\
\text { R: ATTATCGGCCTAACATACATC } \\
\text { P: } \\
\text { /FAM/CCTTCATAT/ZEN/CTATACACATCAAGTTGTT/IA } \\
\text { BkFO/ }\end{array}$ & $\begin{array}{l}\text { IDT } \\
\text { IDT } \\
\text { IDT }\end{array}$ & $\begin{array}{l}\text { RT- } \\
\text { ddPCR }\end{array}$ & 55 & [1] \\
\hline BRSV & $\begin{array}{l}\text { F: GCAATGCTGCAGGACTAGGTATAAT } \\
\text { R: ACACTGTAATTGATGACCCCATTCT } \\
\text { P: } \\
\text { /HEX/ACCAAGACT/ZEN/TGTATGATGCTGCCAAAGCA/I } \\
\text { ABkFO/ }\end{array}$ & $\begin{array}{l}\text { IDT } \\
\text { IDT } \\
\text { IDT }\end{array}$ & $\begin{array}{l}\text { RT- } \\
d d P C R^{a}\end{array}$ & 55 & [3] \\
\hline PMMoV & $\begin{array}{l}\text { F: GAGTGGTTTGACCTTAACGTTGA } \\
\text { R: TTGTCGGTTGCAATGCAAGT } \\
\text { P: FAM/CCTACCGAAGCAAATG/MGBNFQ/ }\end{array}$ & $\begin{array}{l}\text { IDT } \\
\text { IDT } \\
\text { Applied } \\
\text { Biosystems }\end{array}$ & $\begin{array}{l}\text { RT- } \\
\text { ddPCR }^{a}\end{array}$ & 60 & [8] \\
\hline HF183 & $\begin{array}{l}\text { F: ATCATGAGTTCACATGTCCG } \\
\text { R: CGTTACCCCGCCTACTATCTAATG } \\
\text { P: FAM/TCCGGTAGACGATGGGGATGCGTT/MGBNFQ/ }\end{array}$ & $\begin{array}{l}\text { IDT } \\
\text { IDT } \\
\text { Applied } \\
\text { Biosystems }\end{array}$ & $\mathrm{qPCR}^{\mathrm{b}}$ & 60 & [9], [10] \\
\hline
\end{tabular}

${ }^{a}$ TT-ddPCR: Primers and probes at a final concentration of $900 \mathrm{nM}$ and $250 \mathrm{nM}$, respectively.

${ }^{\mathrm{b}} \mathrm{qPCR}$ : Primers and probes at a final concentration of $1 \mu \mathrm{M}$ and $80 \mathrm{nM}$, respectively.

Table S2 Influence of the incubation time ( 0 vs 30 min on a rocker at $4^{\circ} \mathrm{C}$ ) on $\mathrm{BCoV}$ recovery rates and $\mathrm{N} 1$ and $\mathrm{N} 2$ concentrations

\begin{tabular}{lllll}
\hline Sample & $\begin{array}{l}\text { Incubation } \\
\text { time (min) }\end{array}$ & $\begin{array}{l}\text { BCoV recovery } \\
\text { rate (\%) }\end{array}$ & N1 (cp/L) & N2 (cp/L) \\
\hline Green Bay DP & 0 & 7.40 & 12137 & 6058 \\
Sept 7, 2020 & 30 & 4.79 & 14470 & 6878 \\
Milwaukee JI & 0 & 3.51 & 9336 & 2916 \\
Sept 9, 2020 & 30 & 2.21 & 7363 & 1949 \\
Milwaukee SS & 0 & 5.17 & 7423 & 2935 \\
Sept 9, 2020 & 30 & 2.66 & 6898 & 2983 \\
Racine RAC & 0 & 6.29 & 5666 & 5616 \\
Sept 6, 2020 & 30 & 3.37 & 5086 & 3410 \\
\hline
\end{tabular}




\section{Supplemental Figures}

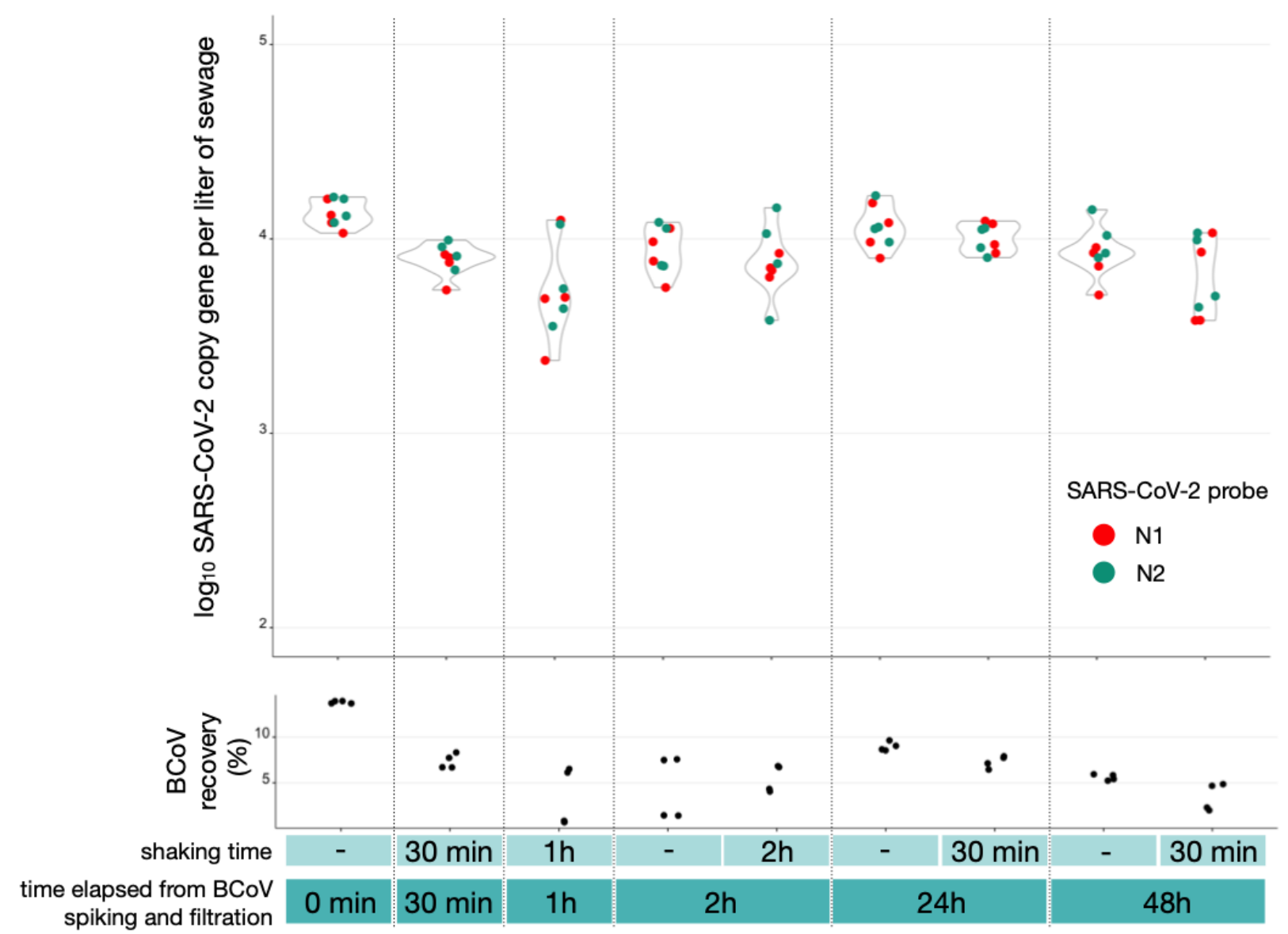

Figure S1 Influence of BCoV incubation time (with or without shaking) on N1/N2 detection and $\mathrm{BCoV}$ recovery on Milwaukee JI influent (Sept 1, 2020). The shaking (on rocker shaker at $4^{\circ} \mathrm{C}$ ) was done at the beginning of the incubation. 

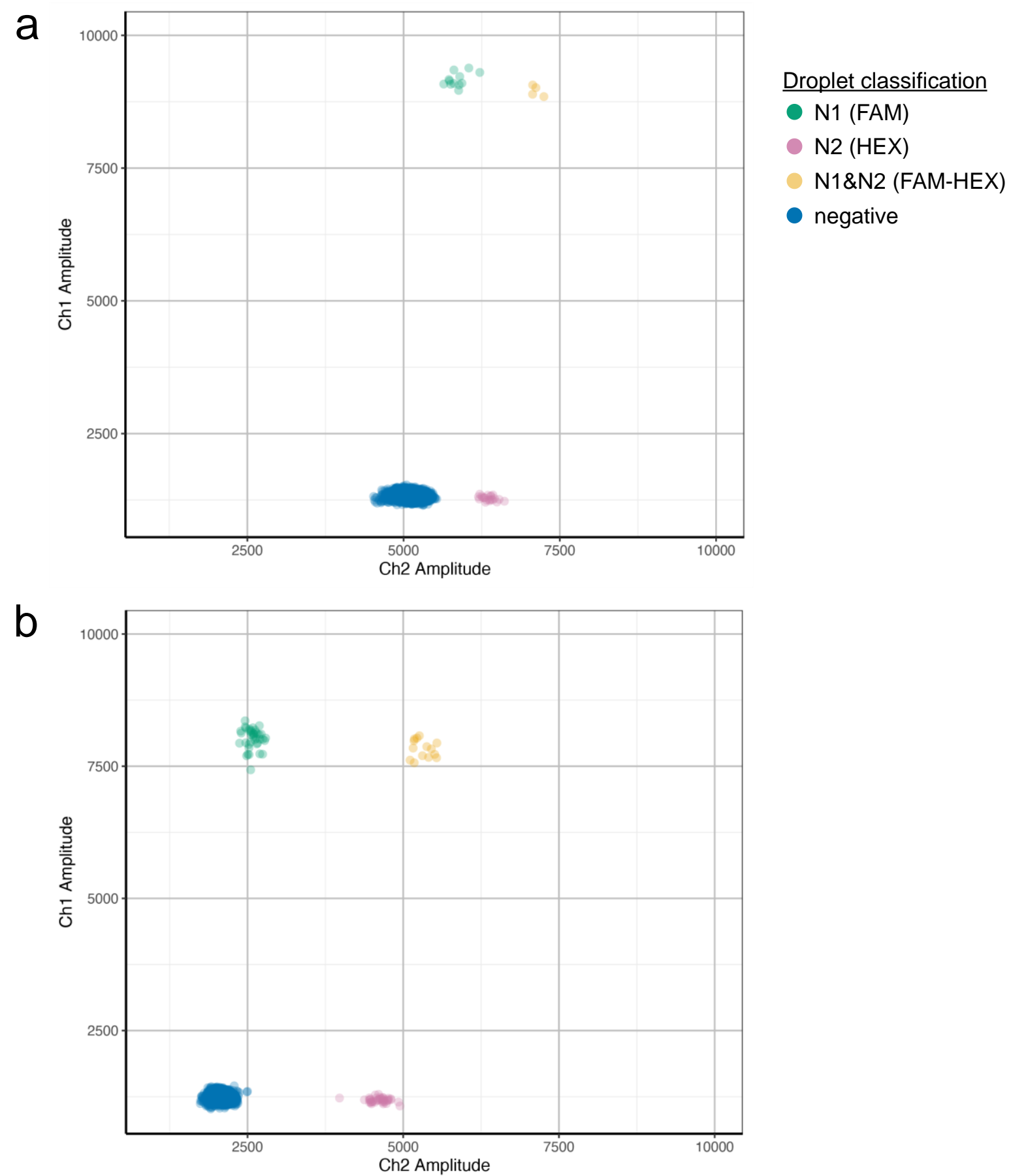

Figure S2 Two-dimensional RT-ddPCR cluster plots of N1/N2 duplex assays using single- and double- quenched N2 probes. Channel 1 amplitude (FAM ${ }^{\mathrm{TM}}$ fluorescent reporter dye) is plotted against Channel $2\left(\mathrm{HEX}^{\mathrm{TM}}\right)$ for (a) the $\mathrm{N} 1$ probe $\left(5^{\prime} \mathrm{FAM}^{\mathrm{TM}} / \mathrm{ZEN}^{\mathrm{TM}} / 3^{\prime} \mathrm{IB}{ }^{\circledR} \mathrm{FQ}\right)$ and single-quenched $\mathrm{N} 2$ probe $\left(5^{\prime} \mathrm{HEX}^{\mathrm{TM}} / 3^{\prime} \mathrm{BHQ}^{\circledR}-1\right)$ duplex assay and $(\mathrm{b})$ the same $\mathrm{N} 1$ probe $\left(5^{\prime} \mathrm{FAM}^{\mathrm{TM}} / \mathrm{ZEN}^{\mathrm{TM}} / 3^{\prime} \mathrm{IB}^{\circledR} \mathrm{FQ}\right.$ and double-quenched $\mathrm{N} 2$ probe $\left(5^{\prime} \mathrm{HEX}^{\mathrm{TM}} / \mathrm{ZEN}^{\mathrm{TM}} / 3^{\prime} \mathrm{IB}^{\circledR} \mathrm{FQ}\right)$ duplex assay. The amplitude between the negative and the N2 droplet clusters was $\sim 1000$ using the single-quenched probe and $\sim 2500$ using the double-quenched probe. 
a

\begin{tabular}{llcccccc}
\hline & Source & Df & $\sigma^{2}$ & $\sigma^{2}$ rel [\%] & $\begin{array}{c}\text { CV [\%] } \\
\mathrm{n}=116\end{array}$ & $F$ & $P$ \\
\hline$(1)$ & WWTP & 5 & 0 & 0.83 & 0.48 & 12.305 & $<0.001$ \\
$(2)$ & Sample & 23 & 0.03 & 52.81 & 3.86 & 25.459 & $<0.001$ \\
$(3)$ & Filter & 29 & 0.01 & 22.45 & 2.52 & 5.229 & $<0.001$ \\
$(4)$ & Assay (error) $\varepsilon$ & 58 & 0.01 & 23.91 & 2.6 & & \\
& Total & 50.78 & 0.05 & 100 & & & \\
\hline
\end{tabular}

$\sigma^{2}=$ variance component

$\sigma^{2}$ rel. = relative variance component (related to the overall variance)

b

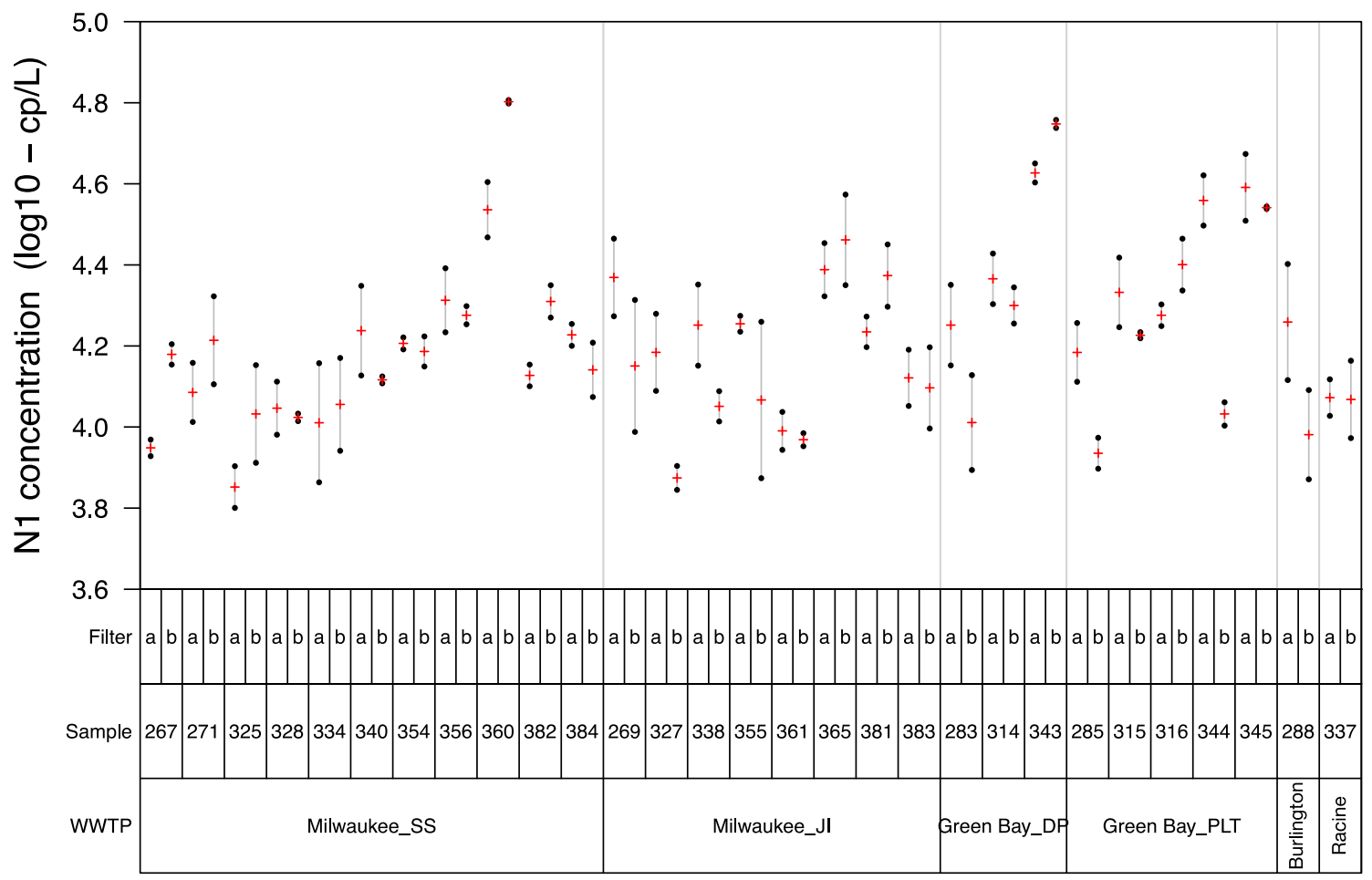

Figure S3 (a) Nested analysis of variance results for assays, filters, samples, and WWTP. (b) Variability chart depicting the analysis. Only samples with $\mathrm{N} 1$ concentrations above the limit of quantification were used. Variance component analysis was performed using the function anovaVCA (package VCA v1.4.3) and the following command anovaVCA ( $\log _{10}$-transformed N1 WWTP/Sample/Filter), $\mathrm{F}$ and $\mathrm{P}$ values were obtained using the function aov and the following command aov ( $\log _{10}$-transformed N1 WWTP/Sample/Filter). 

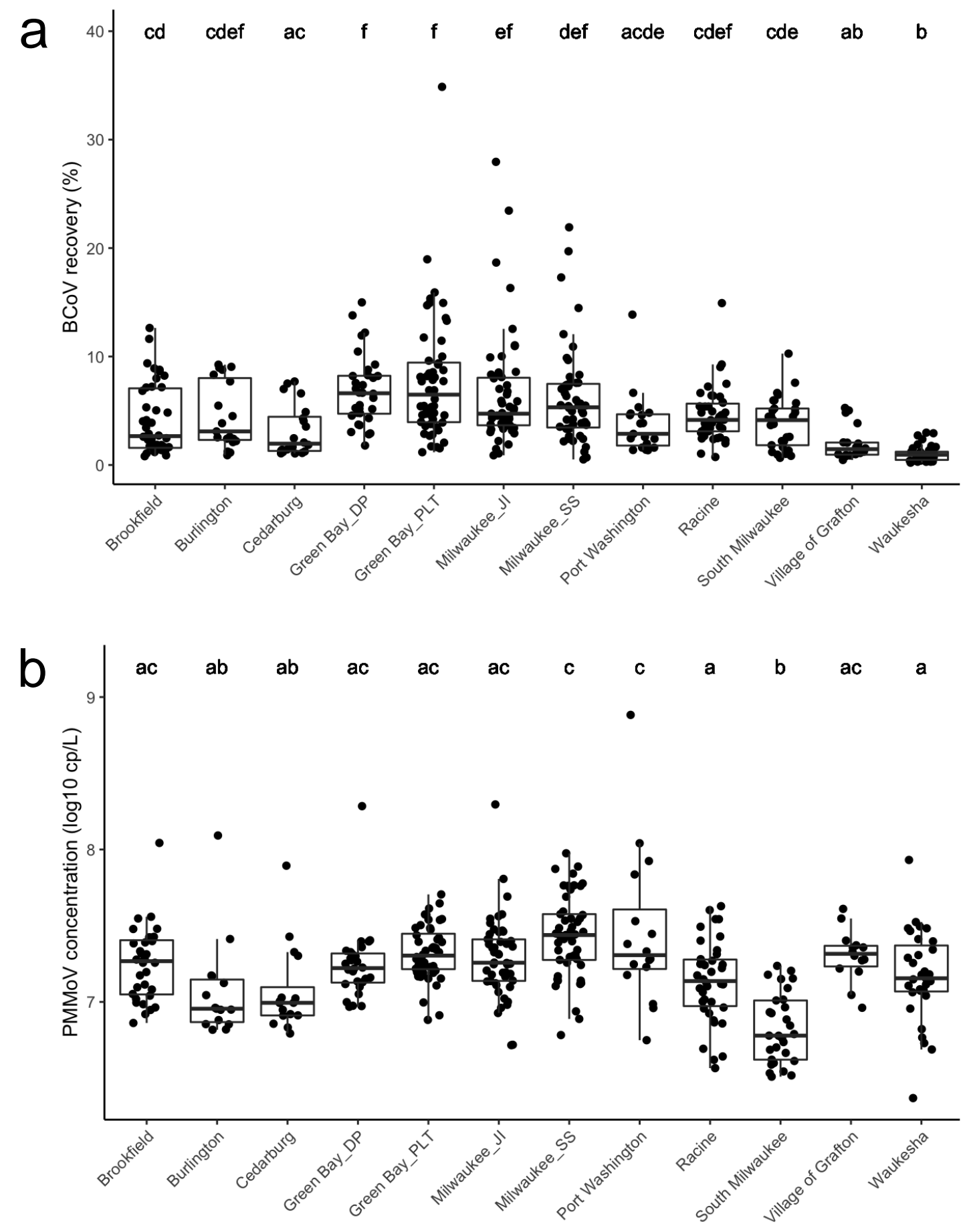

Figure S4 Distribution of (a) BCoV recovery rates and (b) PMMoV concentrations averaged per day and per WWTP. Groups sharing the same letter are not significantly different (ANOVA, BCoV: $F(11)=26.91, P<0.001, \log _{10}$-transformed data; PMMoV: $F(11)=10.76, P<0.001, \log _{10^{-}}$ transformed data; Tukey-adjusted, alpha $=0.05)$. 
a

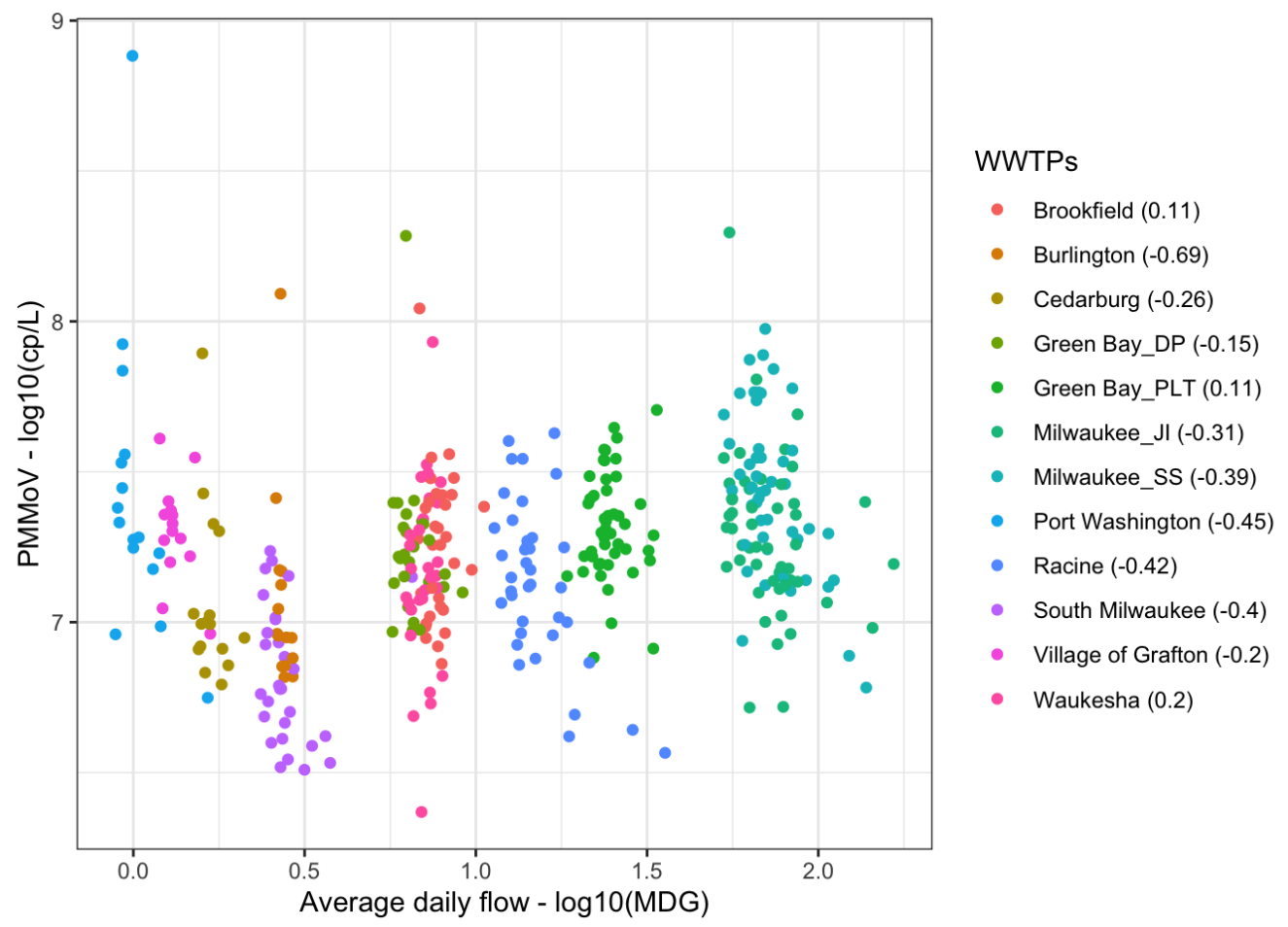

b

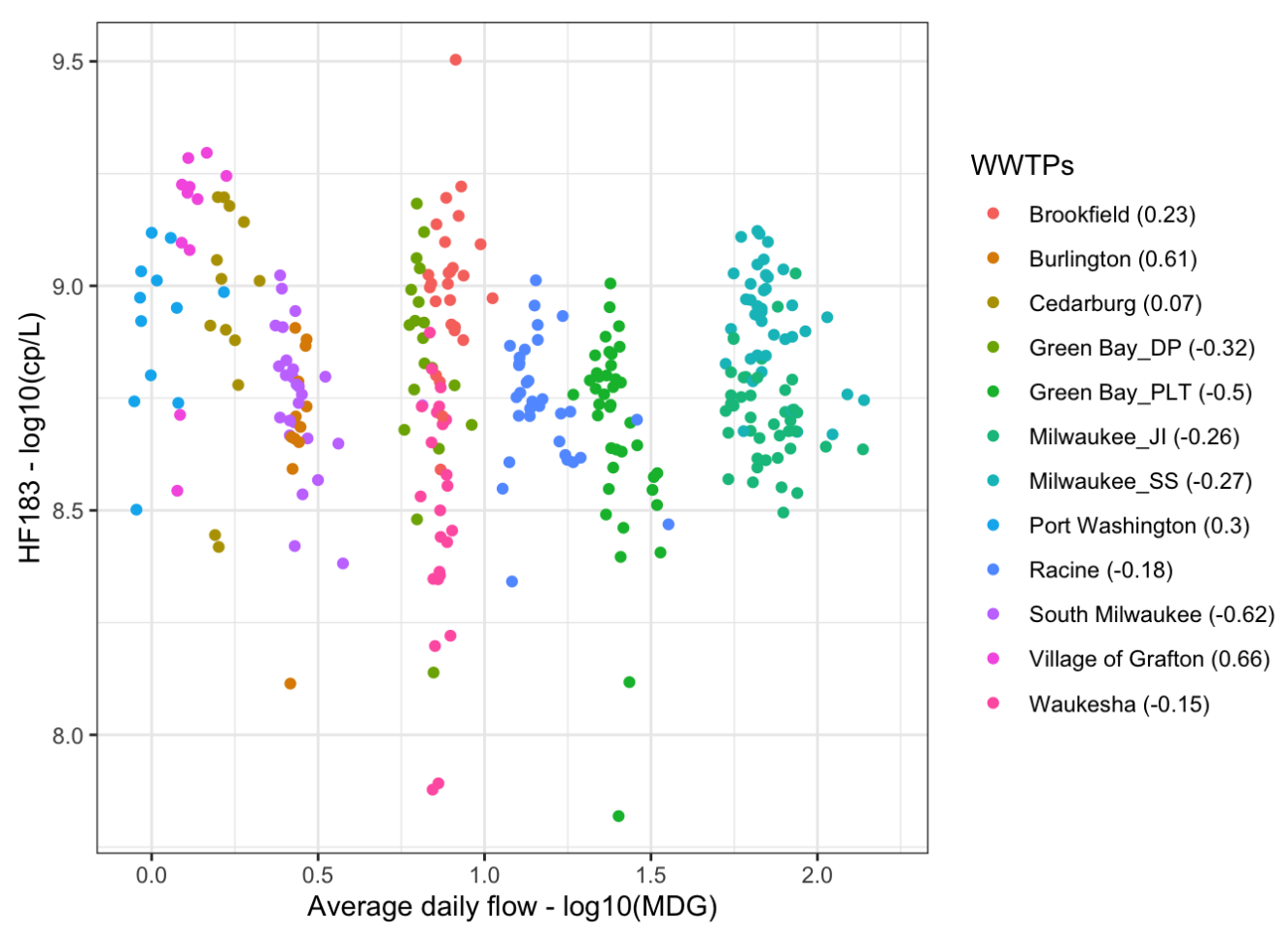

Figure S5 Relationship between the concentrations of human markers (a) PMMoV and (b) HF183 and WWTP average daily flow. Values inside the parentheses display Spearman's rank correlation coefficients. 


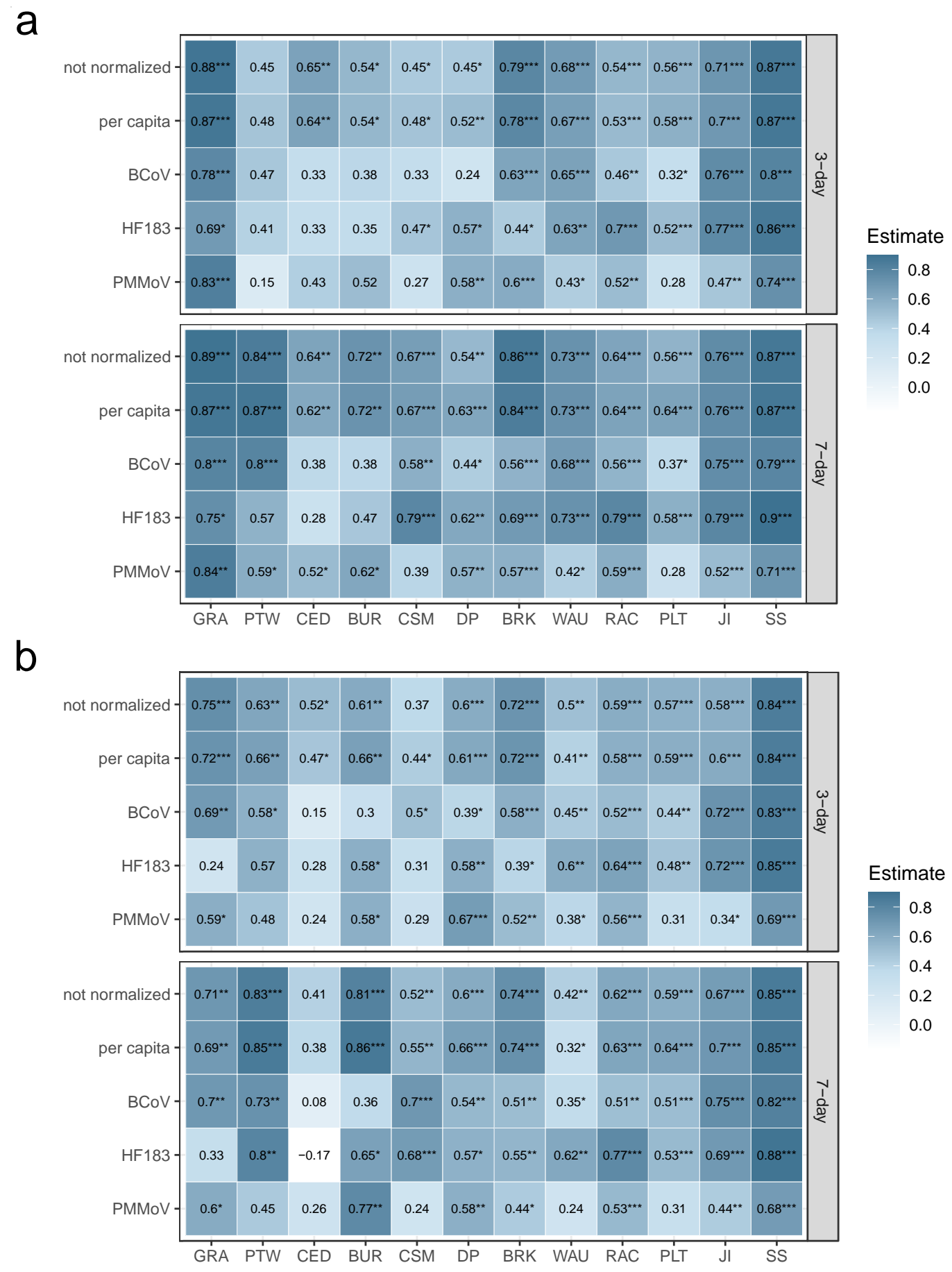

Figure S6 Spearman's rank correlation coefficients between case rate per 100,000 people (using specimen collection date) averaged per 3-day or 7-day and (a) N1 and (b) N2 concentrations in sewage without normalization or normalized to per capita in the served area, BCoV recovery rate, HF183 or PMMoV concentration. Cities are sorted from the smallest population served to the largest. Abbreviations: GRA: Grafton; PTW: Port Washington; CED: Cedarburg; BUR:

Burlington; CSM: City of South Milwaukee; DP: Green Bay De Pere; BRK: Brookfield; WAU: Waukesha; RAC: Racine; PLT: Green Bay; JI: Milwaukee Jones Island; SS: Milwaukee South Shore. P-value significance level: ${ }^{*} 0.05, * * 0.01, * * * 0.001$. 


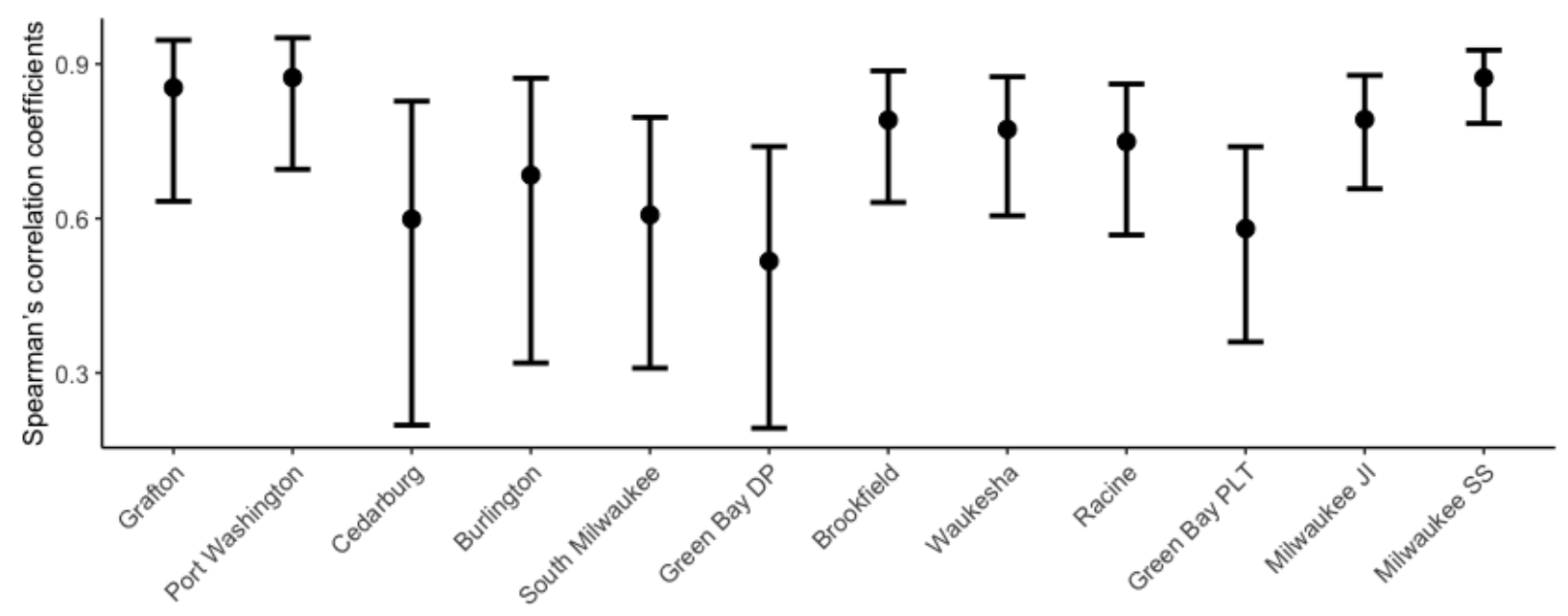

Figure S7 95\% confidence intervals for Spearman's rank coefficients between N1 wastewater concentrations (not normalized) and diagnosed COVID-19 clinical case data (7-day moving average based on the date of specimen collection). Cities are sorted from the smallest population served to the largest. 


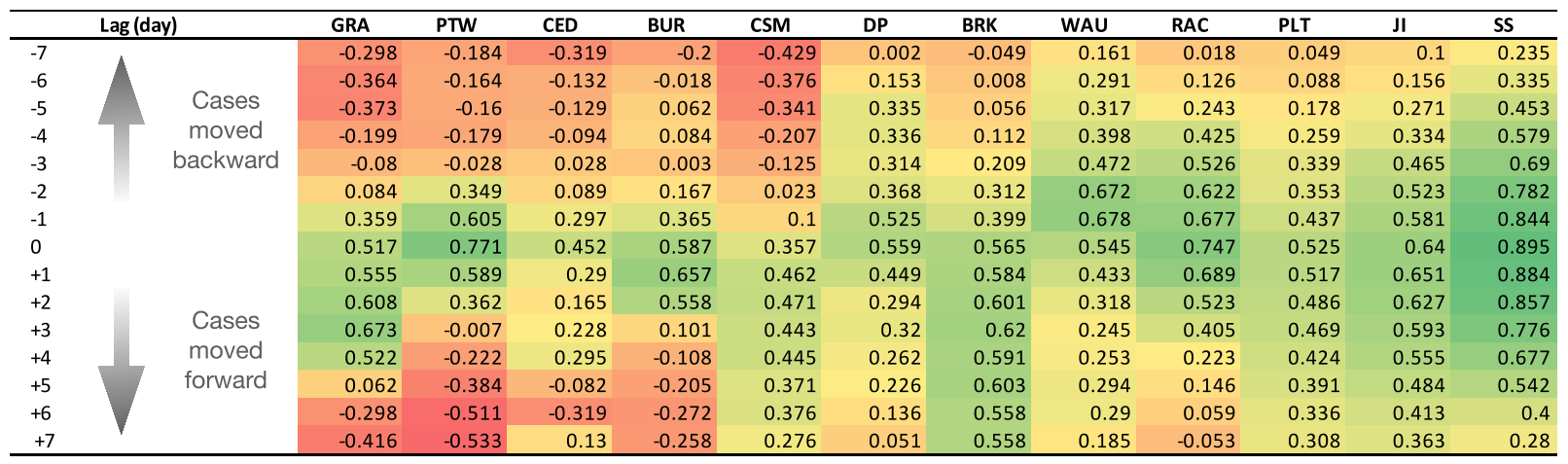

Figure S8 Correlation coefficients for +/- 7 days (lag) coefficients between case rate per 100,000 people (using specimen collection date) and load-normalized SARS-CoV-2 concentrations in sewersheds. Data obtained using cross-correlation functions. Cities are sorted from the smallest population served to the largest. Abbreviations: GRA: Grafton; PTW: Port Washington; CED: Cedarburg; BUR: Burlington; CSM: City of South Milwaukee; DP: Green Bay De Pere; BRK: Brookfield; WAU: Waukesha; RAC: Racine; PLT: Green Bay; Jl: Milwaukee Jones Island; SS: Milwaukee South Shore. 


\section{References}

[1] N. Decaro et al., "Detection of bovine coronavirus using a TaqMan-based real-time RTPCR assay," J. Virol. Methods, vol. 151, no. 2, pp. 167-171, 2008.

[2] D. A. Armbruster and T. Pry, "Limit of blank, limit of detection and limit of quantitation.," Clin. Biochem. Rev., vol. 29 Suppl 1, no. August, pp. S49-52, 2008.

[3] M. Kishimoto et al., "Development of a one-run real-time PCR detection system for pathogens associated with bovine respiratory disease complex," J. Vet. Med. Sci., vol. 79, no. 3, pp. 517-523, 2017.

[4] B. M. Pecson et al., "Reproducibility and sensitivity of 36 methods to quantify the SARSCoV-2 genetic signal in raw wastewater: Findings from an interlaboratory methods evaluation in the U.S.," Environ. Sci. Water Res. Technol., vol. 7, p. 504, 2021.

[5] A. Schuetzenmeister and F. Dufey, "VCA: Variance Component Analysis," R Packag. version 1.4.3, 2020.

[6] A. Signorell, "No Title," DescTools: Tools for Descriptive Statistics. [Online]. Available: https://rdocumentation.org/packages/DescTools/versions/0.99.41. [Accessed: 05-Jul2021].

[7] CDC, "2019-Novel Coronavirus ( 2019-nCoV ) Real-time rRT-PCR Panel Primers and Probes Note," Div. Viral Dis. Centers Dis. Control Prev., 2020.

[8] T. Zhang et al., "RNA viral community in human feces: Prevalence of plant pathogenic viruses," PLoS Biol., vol. 4, no. 1, pp. 0108-0118, 2006.

[9] A. E. Bernhard and K. G. Field, "A PCR Assay To Discriminate Human and Ruminant Feces on the Basis of Host Differences in Bacteroides-Prevotella Genes Encoding 16S rRNA," Appl. Environ. Microbiol., vol. 66, no. 10, pp. 4571-4574, 2000.

[10] H. A. Templar, D. K. Dila, M. J. Bootsma, S. R. Corsi, and S. L. Mclellan, "Quantification of human-associated fecal indicators reveal sewage from urban watersheds as a source of pollution to Lake Michigan," Water Res., vol. 100, pp. 556-567, 2016. 